\title{
Video-assisted thoracic surgery double sleeve bilobectomy of right upper and middle lobes
}

\author{
Jiandong Mei ${ }^{1,2}$, Lin $\mathrm{Ma}^{1,2}$, Qiang Pu ${ }^{1,2}$, Chengwu Liu ${ }^{1,2}$, Lunxu Liu ${ }^{1,2}$ \\ ${ }^{1}$ Department of Thoracic Surgery, West China Hospital, Chengdu 610041, China; ${ }^{2}$ Western China Collaborative Innovation Center for Early \\ Diagnosis and Multidisciplinary Therapy of Lung Cancer, Sichuan University, Chengdu 610041, China \\ Correspondence to: Dr. Lunxu Liu. Department of Thoracic Surgery, West China Hospital, Sichuan University, No. 37, Guoxue Alley, Chengdu \\ 610041, China. Email: lunxu_liu@aliyun.com.
}

Submitted Jul 08, 2018. Accepted for publication Jul 17, 2018.

doi: $10.21037 /$ jtd.2018.07.71

View this article at: http://dx.doi.org/10.21037/jtd.2018.07.71

\section{Introduction}

The evidence on the advantages of minimally invasive approach, represented by video-assisted thoracic surgery (VATS), in surgical treatment of both benign and malignant pulmonary diseases is mountain. Currently, VATS major pulmonary resection is routinely performed in many centers worldwide for patients with non-small cell lung cancer. However, hilar tumor invasion requiring bronchial sleeve or bronchovascular double sleeve lobectomy is generally considered as a contraindication of VATS (1). Even though the VATS bronchial sleeve lobectomy was reported more than one decade ago (2), VATS bronchovascular double sleeve lobectomy remained blank until our first report in 2014 (3). In recent years, a few high-volume centers have tried to perform such operations through the VATS approach (4-7). The results of VATS double sleeve lobectomy in our center were summarized recently (7). Most of the previous reported cases were the operations on the left side. Here we focused on the description of technical aspects of this challenging operation with a more detailed video which depicted a case of VATS bronchovascular double sleeve right upper and middle bilobectomy (Figure 1).

\section{Surgical procedures}

This is a 61-year-old male patient with 60 pack-years of cigarette smoking. He was diagnosed as adenosquamous cell carcinoma involving both the right upper lobar bronchus, main pulmonary artery (PA) and middle lobe. After the systemic assessment, the operation was carried out under general anesthesia with double-lumen intubation. The patient was placed in the right lateral decubitus position with the operation table flexed to a 30-degree angle. A 1-cm long thoracoscopic port was made in the sixth intercostal space (ICS) at the midaxillary line. The other two surgical ports were made in the third and eighth ICS on the anterior axillary line and subscapular line with $3-$ and $2-\mathrm{cm}$ in length, respectively. After thoroughly exploring the chest, the right upper lobe was grasped and pulled posteriorly using the endoscopic ring forceps. The operation started from the anterior of the hilum using an endoscopic suction incorporating with the electrocautery.

The superior pulmonary vein was mobilized first, followed by en bloc removal of the level 2 and level 4 lymph nodes with the non-grasping technique as we previously described (9). The lung was then pulled anteriorly. The posterior mediastinal pleura were incised to remove level 7 lymph nodes. The main structures of the hilum were "hollowed out" till now. We then transected the superior pulmonary vein and the truncus anterior using endo-cutter. Further mobilization of distal PA confirmed that it was invaded by the tumor. The right main PA was mobilized and clamped using a releasable Bulldog clamp (Aesculap, Inc., Center Valley, PA, USA). We continued to incise the oblique fissure and transected the middle lobe vein and bronchus one by one. The common basal artery was then mobilized and clamped with another Bulldog. Further sharp dissection found that the right superior segmental artery was also invaded by the tumor, and was ligated after suturing. The posterior part of oblique fissure was opened with an endo-cutter. After dissecting the surrounding soft 


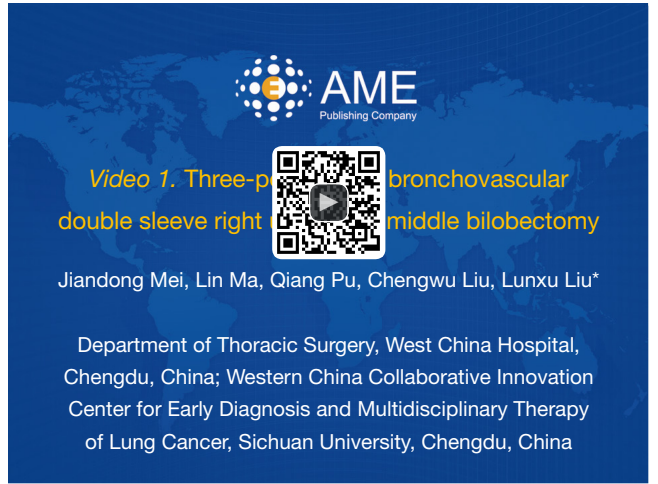

Figure 1 The video depicts a three-portal VATS bronchovascular double sleeve right upper and middle bilobectomy (8). The operation was carried out with the "Hollow-out" process. Lymph nodes and nearby soft tissues of the right hilum and mediastinum were firstly removed to hollow out the main structures of the hilum. The superior pulmonary vein and truncus anterior were transected one by one, followed by clamping the proximal PA using a releasable Bulldog clamp. The anterior part of the oblique fissure was incised, followed by transecting the middle lobe vein and bronchus. The common basal artery was mobilized and clamped with another Bulldog. Bronchial and arterial reconstruction was accomplished using running stiches after removing the diseased lobes. VATS, video-assisted thoracic surgery.

Available online: http://www.asvide.com/article/view/26249

tissues, the intermediate bronchus and right main bronchus were cut with a scalpel and a pair of scissors. Furthermore, the diseased right upper and middle lobes were retrieved using a bag made from surgical glove.

Bronchial reconstruction was carried out firstly with running suture using 3-0 Prolene stiches with two needles. Water-seal test was applied to check for air leak after the anastomosis. PA anastomosis was also performed with running suture using 5-0 Prolene stiches between the common basal artery and the right main PA after rinsing with $0.02 \%$ heparin solution. The operation was finished in $305 \mathrm{~min}$ with $200 \mathrm{~mL}$ of blood loss. The patient recovered well after surgery.

\section{Comments}

In patients with centrally located non-small cell lung cancer involving both the bronchus and PA, bronchovascular double sleeve lobectomy is the more preferred surgical option than pneumonectomy. These patients may have better quality of life after surgery for the preservation of more lung parenchyma than undergoing pneumonectomy. However, surgical procedure requiring bronchovascular reconstruction is usually performed through open thoracotomy. The advantages of VATS encouraged us to expand its application in thoracic surgery. In recent years, we performed several cases of VATS double sleeve lobectomy (7). Most of the reported cases who underwent VATS double sleeve lobectomy were on the left side. The present video depicted the details of this challenging procedure for a case on the right side.

The key technical points of VATS double sleeve lobectomy included the design of surgical approach and procedure, main PA controlling, and bronchovascular reconstruction. The distribution of the incisions was modified from our routine VATS lobectomy (10) to adapt VATS bronchovascular reconstruction. The thoracoscopic port and assistant port were both moved up one ICS on the right side when comparing with our routine VATS lobectomy. This was aimed to achieve better exposure of the hilum during suturing. In addition, an appropriate surgical process was required. We designed the "Hollowout" process for this challenging operation. The important point is to remove the lymphatic tissues and connective tissues surrounding the hilar structures and in the subcarina area to facilitate subsequent isolation of the main PA and bronchus. How to safely control the main PA is another important issue of this operation. With the "Hollow-out" process, the main PA was mobilized without difficulty and enabled us to clamp it with releasable Bulldogs. In Huang's case series, some surgeons used an additional port to place a standard vascular clamp which was used during open surgery (5). This could be avoided with the use of releasable Bulldog clamp without interfering further surgical manipulation.

Bronchovascular reconstruction is the main technical difficulty of VATS double sleeve lobectomy. The right main bronchus is anatomically beneath the main PA on thoracoscopic view. For this reason, the bronchus was reconstructed firstly, which also avoided the tension on more fragile PA when suturing the bronchus. Running suture is the more preferred technique for the anastomosis because of saving time when comparing with interrupted suture. In this video, the anastomosis was performed with the two needle-holder style, which was lately developed and named as the "two-needle-holder suturing technique" (7). Currently, two needle-holders are recommended for the suturing during VATS bronchovascular reconstruction. This is more convenient for the avoiding of exchanging 
surgical instruments between the two operative ports. Both of the needle-holder can be used to do suturing and holding the needle through different directions when necessary.

In conclusion, thoracoscopic bronchovascular double sleeve lobectomy is technically difficult, but feasible. This video demonstrates the practicability of VATS double sleeve lobectomy on the sight side with surgical details.

\section{Acknowledgements}

Funding: This study was supported by the Key Science and Technology Program of Sichuan Province, People's Republic of China (2016FZ0118 to Dr. L Liu).

\section{Footnote}

Conflicts of Interest: The authors have no conflicts of interest to declare.

Informed Consent: Written informed consent was obtained from the patient for publication of this manuscript and any related video.

\section{References}

1. Mulligan MS. Video-Assisted Thoracic Surgery Lobectomy. Oper Tech Thorac Cardiovasc Surg 2012;17:125-42.

2. Santambrogio L, Cioffi U, De Simone M, et al. Video-

Cite this article as: Mei J, Ma L, Pu Q, Liu C, Liu L. Videoassisted thoracic surgery double sleeve bilobectomy of right upper and middle lobes. J Thorac Dis 2018;10(8):5120-5122. doi: $10.21037 /$ jtd.2018.07.71
Assisted Sleeve Lobectomy for Mucoepidermoid Carcinoma of the Left Lower Lobar Bronchus: A Case Report. Chest 2002;121:635-6.

3. Liu L, Mei J, Pu Q, et al. Thoracoscopic bronchovascular double sleeve lobectomy for non-small-cell lung cancer. Eur J Cardiothorac Surg 2014;46:493-5.

4. Gonzalez-Rivas D, Delgado M, Fieira E, et al. Double sleeve uniportal video-assisted thoracoscopic lobectomy for non-small cell lung cancer. Ann Cardiothorac Surg 2014;3:E2.

5. Huang J, Li J, Qiu Y, et al. Thoracoscopic double sleeve lobectomy in 13 patients: a series report from multicenters. J Thorac Dis 2015;7:834-42.

6. Qiu T, Zhao Y, Xuan Y, et al. Robotic-assisted doublesleeve lobectomy. J Thorac Dis 2017;9:E21-E25.

7. Mei J, Guo C, Pu Q, et al. Video-assisted thoracic surgery double sleeve lobectomy for non- small cell lung cancer: a report of seven cases. Video-assist Thorac Surg 2018;3:1.

8. Mei J, Ma L, Pu Q, et al. Three-portal VATS bronchovascular double sleeve right upper and middle bilobectomy. Asvide 2018;5:662. Available online: http:// www.asvide.com/article/view/26249

9. Liu C, Pu Q, Guo C, et al. Non-grasping en bloc mediastinal lymph node dissection for video-assisted thoracoscopic lung cancer surgery. BMC Surgery 2015:38.

10. Liu L, Che G, Pu Q, et al. A new concept of endoscopic lung cancer resection: Single-direction thoracoscopic lobectomy. Surg Oncol 2010;19:e71-7. 